\title{
THERMODYNAMIC ANALYSIS OF THE OSCILLATORY CONVECTIVE INSTABILITY IN HOMEOTROPIC NEMATICS HEATED FROM BELOW
}

\author{
H. N. W. LEKKERKERKER
}

Faculteit van de Wetenschappen Vrije Universiteit Brussel, 1050 Brussel, Belgium

\begin{abstract}
Résumé. - Nous présentons une analyse thermodynamique de l'instabilité convective oscillante dans des nématiques homéotropes, chauffés par le bas. Cette analyse montre que l'instabilité oscillante se produit à partir de la compétition entre un effet stabilisant à long temps de relaxation et un effet déstabilisant à temps de relaxation court. L'effet d'un champ magnétique stabilisant est aussi pris en considération.
\end{abstract}

\begin{abstract}
A thermodynamic analysis of the recently discovered oscillatory convective instability in homeotropic nematics heated from below is presented. From this analysis it follows that the oscillatory convective instability arises from the competition between a stabilizing effect with a long relaxation time and a destabilizing effect with a short relaxation time. The effect of a stabilizing magnetic field is also considered.
\end{abstract}

1. Introduction. - Recently we predicted that a homeotropic nematic heated from below would become unstable to oscillatory convection [1]. This theoretical prediction has been experimentally confirmed by Guyon, Pieranski and Salan [2]. They also studied the effect of a stabilizing magnetic field both experimentally as well as theoretically.

In reference [1] we pointed out that the oscillatory convection arises from the competition between a stabilizing effect with a long relaxation time $\tau_{\text {stab }}$ and a destabilizing effect with a shorter relaxation time $\tau_{\text {destab}}$. The idea being that a velocity perturbation with an oscillation frequency $\tau_{\text {stab }}^{-1}<\omega<\tau_{\text {destab }}^{-1}$ effectively eliminates the stabilizing effect by putting it out of phase while retaining the destabilizing effect. On the basis of this argument one expects that the oscillatory instability will set in at roughly the same threshold as that for which stationary convection would occur in the absence of the stabilizing effect.

These ideas are schematically illustrated in figure 1 for the case of a homeotropic nematic with a positive heat diffusion anisotropy $\left(\chi_{\mathrm{a}}=\chi_{\|}-\chi_{\perp}>0, \chi_{\|}, \chi_{\perp}\right.$ heat diffusion constant parallel and perpendicular to the director $\mathbf{n}$ ). We consider a velocity field in the form of horizontal rolls with $q_{x} \simeq q_{z}=\pi / d$ and frequency $K \xi q^{2} \ll \omega<\chi q^{2} \quad\left(K\right.$ : elastic constant, $\xi=\gamma_{1}^{-1}$ : director relaxation constant). Due to the sheardirector coupling the flow gives rise to a distortion of the alignment that since $\omega \gg K \xi q^{2}$ has a $90^{\circ}$ phase lag. The result is that the stabilizing influence of the flow distortion induced heat focusing is virtually eliminated.

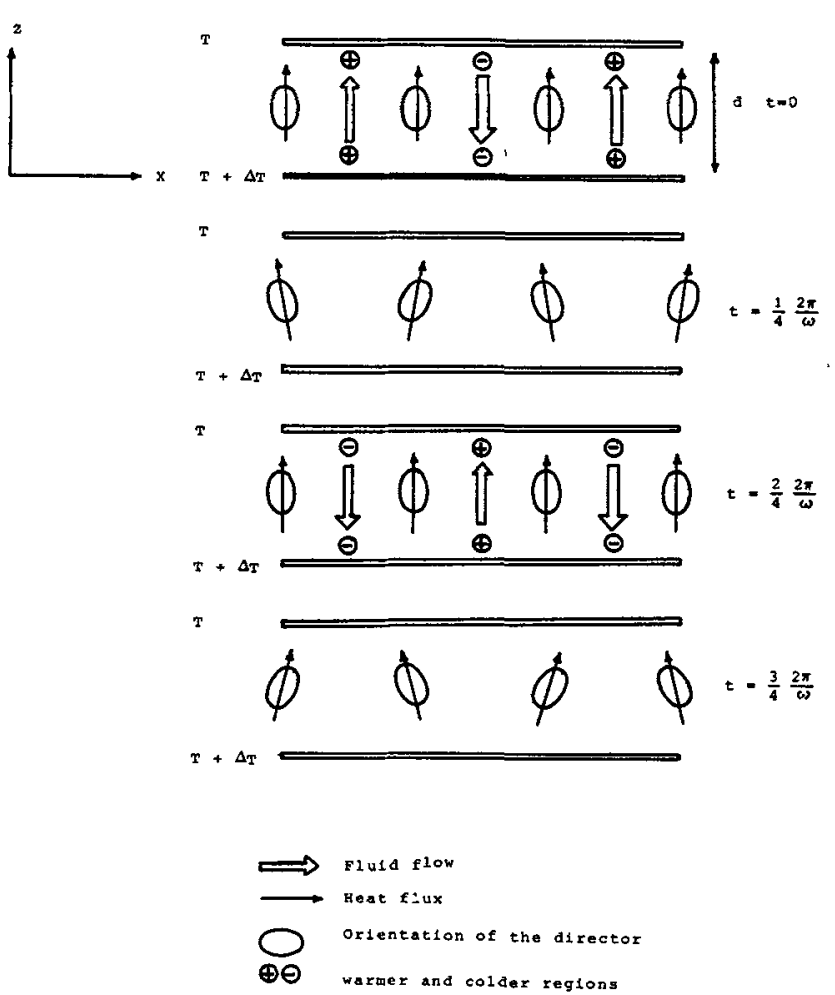

Fig. 1. - Schematic illustration of the mechanism of the oscillatory convective instability in a homeotropic nematic with positive heat diffusion anisotropy heated from below.

It is the purpose of the present work to put the qualitative analysis given above about the nature of the oscillatory convective instability on a quantitative 
basis by using a thermodynamic principle due to Chandrasekhar [3] according to which « The onset of thermal instability will be as overstable oscillations if it is possible (at a lower adverse temperature gradient than necessary for the onset of stationary convection) to balance in a synchronous manner the periodically varying amounts of kinetic and other forms of energy with similarly varying rates of dissipation and liberation of energy ".

2. Stationary convection. - As a preliminary of the analysis of oscillatory convection we first consider the onset of stationary convection in a homeotropic nematic [4-8]. Stationary convection sets in at the minimum temperature gradient at which a steady balance can be maintained between the rate of dissipation of energy and the rate of liberation of thermodynamically available energy by the buoyancy force acting on the fluid. Consider the following stationary velocity perturbation

$$
\begin{aligned}
& v_{x}(\mathbf{r})=-2 \frac{q_{z}}{q_{x}} v_{z}(\mathbf{q}) \sin q_{x} x \cos q_{z} z \\
& v_{y}(\mathbf{r})=0 \\
& v_{z}(\mathbf{r})=2 v_{z}(\mathbf{q}) \cos q_{x} x \sin q_{z} z .
\end{aligned}
$$

Here $q_{z}=\pi / d$, with $d$ the thickness of the homeotropic layer.

The velocity field is chosen such that $\operatorname{div} \mathbf{v}=0$ and $(\operatorname{curl} \mathbf{v})_{z}=0$ since these variables do not play a role in the instability. The rate of viscous dissipation of kinetic energy per unit volume is given by

$$
\dot{F}_{\text {kin }}^{v}=-\frac{1}{V} \int\left(2 v_{1} A_{z z}^{2}+4 v_{3} A_{z x}^{2}+2 v_{2} A_{x x}^{2}\right) \mathrm{d} V .
$$

Here

$$
A_{i j}=\frac{1}{2}\left(\frac{\partial v_{i}}{\partial x_{j}}+\frac{\partial v_{j}}{\partial x_{i}}\right)
$$

and $v_{1}, v_{2}$ and $v_{3}$ are viscosity coefficients.

Substituting the velocity field given by (1) in (2) one obtains

$$
\dot{F}_{\text {kin }}^{v}=-\frac{v q^{4}}{q_{x}^{2}} v_{z}^{2}(\mathbf{q})
$$

Here

$$
v=\frac{1}{2}\left(v_{1}+v_{2}\right) \sin ^{2} 2 \varphi+v_{3} \cos ^{2} 2 \varphi
$$

where $\varphi$ is the angle between $\mathbf{n}^{0}$ and $\mathbf{q}$.

In addition to dissipation of kinetic energy there is also dissipation of elastic energy, which per unit volume is given by

$$
\dot{F}_{\text {elas }}^{d}=-\frac{1}{V} \int \xi h_{x}^{2} \mathrm{~d} V .
$$

Here $h_{x}$ is the thermodynamic force conjugate to the director perturbation $n_{x}$

$$
h_{x}=K_{1} \frac{\partial^{2} n_{x}}{\partial x^{2}}+K_{3} \frac{\partial^{2} n_{x}}{\partial z^{2}}
$$

where $K_{1}$ and $K_{3}$ are the Frank elastic constants for splay and bend deformations. In order to evaluate the elastic dissipation we have to calculate the distortion in the alignment caused by the fluid flow from the equation of motion of the director

$$
\dot{n}_{x}=\frac{\lambda+1}{2} \frac{\partial v_{x}}{\partial z}+\frac{\lambda-1}{2} \frac{\partial v_{z}}{\partial x}+\xi h_{x}
$$

where $\lambda$ is the shear director coupling constant. For a stationary state $\dot{n}_{x}=0$ and one obtains

$$
n_{x}(\mathbf{r})=2 n_{x}(\mathbf{q}) \sin q_{x} x \sin q_{z} z
$$

where the Fourier amplitude $n_{x}(\mathbf{q})$ is given by

with

$$
n_{x}(\mathbf{q})=\frac{C}{K \xi q_{x}} v_{z}(\mathbf{q})
$$

and

$$
C=\frac{1}{2}(1+\lambda \cos 2 \varphi)
$$

$$
K=K_{1} \sin ^{2} \varphi+K_{3} \cos ^{2} \varphi .
$$

Substituting (7) in (4) one obtains

$$
\dot{F}_{\text {elas }}^{d}=-\frac{C^{2} q^{4}}{\xi q_{x}^{2}} v_{z}^{2}(q)
$$

The rate of transfer energy from the gravitational field to the fluid flow per unit volume is given by

$$
\dot{F}_{\text {kin }}^{g}=-\frac{1}{V} g \int \delta \rho v_{z} \mathrm{~d} V
$$

where $g$ is the gravitation constant and $\delta \rho$ is the perturbation in the steady state density distribution, which can be written as

$$
\delta \rho=-\rho \alpha \theta .
$$

Here $\theta$ is the perturbation in the steady state temperature and $\alpha$ is the thermal expansivity. For the temperature perturbation one can write the following (linearized) equation

$$
\dot{\theta}=\beta v_{z}-\chi_{\mathrm{a}} \beta \frac{\partial n_{x}}{\partial x}+\chi_{\perp}\left(\frac{\partial^{2} \theta}{\partial x^{2}}+\frac{\partial^{2} \theta}{\partial y^{2}}\right)+\chi_{\|} \frac{\partial^{2} \theta}{\partial z^{2}} .
$$

In (10) the first term on the r.h.s. takes into account convective heat transport in a vertical temperature gradient $\beta=-\partial T / \partial z$, the second term represents the effect of heat focusing due to the distortion in the alignment and the third term accounts for diffusive heat transport. Substituting the velocity field given by (1) and the director perturbation given by (7) in (10) one obtains in the stationary state $(\dot{\theta}=0)$

$$
\theta(\mathbf{r})=2 \theta(\mathbf{q}) \cos q_{x} x \sin q_{z} z
$$

where the Fourier amplitude $\theta(\mathbf{q})$ is given by

$$
\theta(\mathbf{q})=\frac{(1-A) \beta}{\chi q^{2}} v_{z}(\mathbf{q})
$$


with

and

$$
A=\frac{C \chi_{\mathrm{a}}}{K \xi}
$$

$$
\chi=\chi_{\|} \cos ^{2} \varphi+\chi_{\perp} \sin ^{2} \varphi .
$$

Using the expression (11) for the temperature perturbation one obtains for the rate of production of kinetic energy by the buoyancy force

$$
\dot{F}_{\text {kin }}^{g}=\frac{\rho g \alpha \beta(1-A)}{\chi q^{2}} v_{z}^{2}(\mathbf{q}) .
$$

For MBBA the parameter $A \simeq 300$ and thus the heat focusing effect dominates the contribution of heat transport by convection. The result is that for a homeotropic nematic with a positive heat diffusion anisotropy there is only a positive production of kinetic energy and thus the possibility of a stationary convective instability if one heats from above (i.e. $\beta$ negative).

Convection will set in when the rate of dissipation of kinetic energy and elastic energy is balanced by the rate of production of kinetic energy by the buoyancy force

$$
\dot{F}_{\text {kin }}^{v}+\dot{F}_{\text {elas }}^{d}+\dot{F}_{\text {kin }}^{g}=0 .
$$

Substituting (3), (8) and (12) in (13) one obtains

$$
g \alpha \beta_{\mathrm{c}}^{\mathrm{s}} d^{4}=\frac{\left(\frac{v}{\rho}+\frac{C^{2}}{\rho \xi}\right) \chi}{1-A} \frac{q^{6} d^{4}}{q_{x}^{2}} .
$$

This result is in agreement with previous work [4-8]. The advantage of the analysis presented here is that it allows one to give a precise physical meaning of the various contributions that appear in the numerator and denominator of the expression at the r.h.s. of (14).

3. Oscillatory convection. - Consider a velocity field with the same spatial dependence as the velocity field given by (1) and oscillating with a frequency $K \xi q^{2} \ll \omega<\chi q^{2}$

$$
\begin{aligned}
& v_{x}(\mathbf{r}, t)=-2 \frac{q_{z}}{q_{x}} v_{z}(\mathbf{q}) \cos \omega t \sin q_{x} x \cos q_{z} z \\
& v_{y}(\mathbf{r}, t)=0 \\
& v_{z}(\mathbf{r}, t)=2 v_{z}(\mathbf{q}) \cos \omega t \cos q_{x} x \sin q_{z} z .
\end{aligned}
$$

We now want to check whether it is possible to balance in a synchronous manner the periodically varying amounts of kinetic and elastic energy with similarly varying amounts of dissipation of kinetic and elastic energy and production of kinetic energy by the buoyancy force, which is the condition for the appearance of oscillatory convection

$$
\dot{F}_{\text {kin }}^{v}+\dot{F}_{\text {elas }}^{d}+\dot{F}_{\text {kin }}^{g}=\dot{F}_{\text {kin }}^{0}+\dot{F}_{\text {elas }}^{0} .
$$

Here $\dot{F}_{\text {kin }}^{0}$ and $\dot{F}_{\text {elas }}^{0}$ are the rates of change of kinetic and elastic energy due to the oscillatory nature of the velocity field and the distortion of alignment. A simple calculation shows that for the frequencies we are considering here that $\dot{F}_{\text {elas }}^{d}, \dot{F}_{\text {elas }}^{0}$ and $\dot{F}_{\text {kin }}^{0}$ are very small compared to $\dot{F}_{\text {kin }}^{v}$ and $\dot{F}_{\text {kin }}^{g}$ and may be neglected in (16).

The viscous dissipation of kinetic energy is now given by

$$
\dot{F}_{\text {kin }}^{v}=-\frac{v q^{4}}{q_{x}^{2}} \cos ^{2} \omega t v_{z}^{2}(\mathbf{q})
$$

In order to calculate the rate of production of kinetic energy by the buoyancy force we have to know the temperature perturbation which can be obtained from the heat eq. (10). However in order to solve that equation we have to know the director perturbation which can be obtained from (6). Substituting the velocity field given by (15) in (6) and taking into account that $\omega \gg K \xi q^{2}$ one obtains

$n_{x}(\mathbf{r}, t) \simeq \frac{1}{\omega} \frac{C q^{2}}{q_{x}} 2 v_{z}(q) \sin \omega t \sin q_{x} x \sin q_{z} z$.

Now solving the heat equations one obtains

$$
\begin{aligned}
\theta(\mathbf{r}, t)=(B \cos \omega t+ & D \sin \omega t) \times \\
& \times 2 v_{z}(\mathbf{q}) \cos q_{x} x \sin q_{z} z
\end{aligned}
$$

with

$$
\begin{aligned}
& B=\frac{\tau_{\theta}^{-1} \beta}{\tau_{\theta}^{-2}+\omega^{2}}+\frac{C \chi_{\mathrm{a}} q^{2} \beta}{\tau_{\theta}^{-2}+\omega^{2}} \\
& D=\frac{\omega \beta}{\tau_{\theta}^{-2}+\omega^{2}}-\frac{C \chi \chi_{\mathrm{a}} q^{4} \beta}{\omega} \\
& \tau_{\theta}^{-2}+\omega^{2}
\end{aligned}
$$

Here $\tau_{\theta}=\left(\chi q^{2}\right)^{-1}$ (thermal relaxation time). The first term in $B$ and $D$ is due to the convective term in the heat equation and the second term arises from the heat focusing effect. Using the expression (19) for the temperature perturbation one obtains for the rate of production of kinetic energy by the buoyancy force

$\dot{F}_{\text {kin }}^{g}=\rho g \alpha\left(B \cos ^{2} \omega t+D \sin \omega t \cos \omega t\right) v_{z}^{2}(\mathbf{q})$.

Substituting (17) and (20) in (16) and balancing the $\cos ^{2} \omega t$ terms one obtains

$$
v \frac{q^{4}}{q_{x}^{2}}+\rho g \alpha B=0
$$

and balancing the $\cos \omega t \sin \omega t$ terms one gets

$$
D=0 \text {. }
$$

From the last condition we immediately get

$$
\omega_{\mathrm{c}}^{0}=\sqrt{C \chi_{\mathrm{a}} \chi} q^{2} \text {. }
$$

We see that indeed $K \xi q^{2} \ll \omega_{\mathrm{c}}<\chi q^{2}$. For a homeotropic layer of MBBA of $5 \mathrm{~mm}$ thick (23) predicts an 
oscillation period $t_{\mathrm{c}}=2 \pi / \omega_{\mathrm{c}} \simeq 200$ seconds. Substituting the oscillation frequency given by (23) in (21) one obtains

$$
g \alpha \beta_{\mathrm{c}}^{0} d^{4}=\frac{v}{\dot{\rho}} \cdot \chi \frac{q^{6} d^{4}}{q_{x}^{2}} .
$$

Thus as predicted on the basis of qualitative considerations one finds that a homeotropic nematic heated from below becomes unstable to oscillatory convection at roughly the same threshold as that for which an isotropic fluid with viscosity $v$ and heat diffusivity $\chi$ becomes unstable to stationary convection.

4. Effect of a stabilizing magnetic field. - As mentioned in the introduction, Guyon, Pieranski and Salan [2] studied the effect of a stabilizing magnetic field both experimentally and theoretically. They put forward a simple one dimensional model which gives a good description of the main physical effects that occur when one applies a stabilizing magnetic field. Here we analyze the effect of a magnetic field using the thermodynamic method used in the previous sections.

If one applies a magnetic field parallel to the director the force conjugate to the director perturbation $n_{x}$ takes the form

$$
h_{x}=K_{1} \frac{\partial^{2} n_{x}}{\partial x^{2}}+K_{3} \frac{\partial^{2} n_{x}}{\partial z^{2}}-\Delta \chi_{\mathrm{m}} H^{2}
$$

$\Delta \chi_{m}$ is the anisotropy of the magnetic susceptibility which is positive in the usual nematics and thus a magnetic field parallel to the director has a stabilizing influence. In the stationary case the magnetic field does not affect the rate of dissipation of kinetic and elastic energy but has a strong influence on the heat focusing effect. Indeed a stabilizing magnetic field reduces the distortion in the alignment caused by the fluid flow. The result is that the factor $A$ that appears in the Fourier amplitude $\theta(\mathbf{q})$ of the temperature perturbation (eq. (11)) in the presence of a stabilizing magnetic field is given by

$$
A=\frac{C \chi_{\mathrm{a}} q^{2}}{\left(K q^{2}+\Delta \chi_{\mathrm{m}} H^{2}\right) \xi} \simeq \frac{C \chi_{\mathrm{a}} q^{2}}{\Delta \chi_{\mathrm{m}} H^{2} \xi}
$$

where the expression on the extreme right is valid for fields larger than $50 \mathrm{G}$. Introducing the dimension less parameters

$$
\begin{aligned}
& a=\frac{C \chi_{\mathrm{a}}}{\chi} \\
& b=\frac{\Delta \chi_{\mathrm{m}} H^{2} \xi}{\chi q^{2}} \\
& c=\frac{C^{2}}{v \xi}
\end{aligned}
$$

the threshold for stationary convection can be written as

$$
g \alpha \beta_{\mathrm{c}}^{\mathrm{s}} d^{4} \simeq \frac{(1+c) b}{b-a} \frac{v}{\rho} \chi \frac{q^{6} d^{4}}{q_{x}^{2}} .
$$

From this expression it follows that for $b>a$ stationary convection when heated from below becomes possible. For a homeotropic layer of MBBA of $5 \mathrm{~mm}$ thickness the point $a=b$ corresponds to a magnetic field of about 180 gauss. For large magnetic fields the heat focusing effect is practically completely eliminated and the threshold reaches its asymptotic value

$$
g \propto \beta_{\mathrm{c}}^{\mathrm{s}} d^{4}=\left(\frac{v}{\rho}+\frac{C^{2}}{\rho \xi}\right) \chi \frac{q^{6} d^{4}}{q_{x}^{2}} .
$$

Note that this asymptotic value differs from the threshold for the onset of oscillatory convection in zero field (eq. (24)) by the presence of a term which accounts for the dissipation of elastic energy. For MBBA

$$
\beta_{\mathrm{c}}^{\mathrm{s}}(H \rightarrow \infty) \simeq 1.4 \beta_{\mathrm{c}}^{0} \quad(H=0) .
$$

The effect of a stabilizing field on the oscillatory instability is very interesting. The relaxation time of director perturbations is now given by

$$
\tau_{n}=\left[\left(K q^{2}+\Delta \chi_{\mathrm{m}} H^{2}\right) \xi\right]^{-1} \simeq\left(\Delta \chi_{\mathrm{m}} H^{2} \xi\right)^{-1} .
$$

It is possible to lower the relaxation time of the long wavelength director perturbation that play a role in the convective instability by several orders of magnitude such that for a certain field strength it becomes comparable to the relaxation time of temperature perturbations. Under these conditions the dephasing between the stabilizing heat focusing effect and the destabilizing convective heat transport term, necessary for the appearance of oscillatory convection, is no longer possible and one expects a change from oscillatory to stationary convection. Again these qualitative arguments can be made quantitative using the thermodynamic method of analysis.

Due to the fact that in the presence of a stabilizing magnetic field the director perturbations relax faster they will no longer be completely $90^{\circ}$ behind in phase with respect to the velocity field. Solving (6) with the velocity field given by (15) and the thermodynamic force $h_{x}$ given by (25) one obtains

$$
n_{x}(\mathbf{r}, t)=2 n_{x}(q, t) \sin q_{x} x \sin q_{z} z
$$

where the Fourier amplitude $n_{x}(\mathbf{q}, t)$ is given by

$$
\begin{aligned}
n_{x}(\mathbf{q}, t)=\left[\frac{\tau_{n}^{-1}}{\tau_{n}^{-2}+\omega^{2}} \frac{C q^{2}}{q_{x}} \cos \omega t+\right. \\
\left.+\frac{\omega}{\tau_{n}^{-2}+\omega^{2}} \frac{C q^{2}}{q_{x}} \sin \omega t\right] v_{z}(\mathbf{q}) .
\end{aligned}
$$


If one now solves the heat equation (10) with the velocity field (15) and the director perturbation (31) one obtains a temperature perturbation of the form (19) with coefficient $B$ and $D$ given by

$$
B=\frac{\tau_{\theta}^{-1} \beta}{\tau_{\theta}^{-2}+\omega^{2}}+\frac{\left(\omega^{2}-\tau_{\theta}^{-1} \tau_{n}^{-1}\right) C \chi_{\mathrm{a}} q^{2} \beta}{\left(\tau_{\theta}^{-2}+\omega^{2}\right)\left(\tau_{n}^{-2}+\omega^{2}\right)}
$$

and

$$
D=\frac{\omega \beta}{\tau_{\theta}^{-2}+\omega^{2}}-\frac{\omega\left(\tau_{\theta}^{-1}+\tau_{n}^{-1}\right) C \chi_{\mathrm{a}} q^{2} \beta}{\left(\tau_{\theta}^{-2}+\omega^{2}\right)\left(\tau_{n}^{-2}+\omega^{2}\right)} .
$$

Again the first term in $B$ and $D$ is due to the convective term in the heat equation and the second term arises from the heat focusing effect. Solving the balance equations (21) and (22) with the above values of $B$ and $D$ one obtains

$$
\omega_{\mathrm{c}}^{2}=\left(\chi q^{2}+\Delta \chi_{\mathrm{m}} H^{2} \xi\right) C \chi_{\mathrm{a}} q^{2}-\left(\Delta \chi_{\mathrm{m}} H^{2} \xi\right)^{2}
$$

and

$$
g \alpha \beta_{\mathrm{c}}^{0} d^{4}=\frac{v}{\rho}\left(\chi q^{2}+\Delta \chi_{\mathrm{m}} H^{2} \xi\right) \frac{q^{4} d^{4}}{q_{x}^{2}} .
$$

From the above equations it follows that $\omega_{c}$ as a function of $H^{2}$ first increases goes through a maximum and then becomes zero and that $\beta_{\mathrm{c}}^{0}$ increases linearly with $H^{2}$. Actually in the presence of a magnetic field the dissipation and oscillation of elastic energy are no longer negligible. Taking them into account and solving the balance equations one obtains for the oscillation frequency and threshold

$$
\begin{array}{r}
\omega_{\mathrm{c}}^{2}=\left[1+\left(\frac{a+c+a c}{a}\right) b-\left(\frac{1+c}{a}\right) b^{2}\right] \times \\
\times C \chi_{\mathrm{a}} \chi q^{4}
\end{array}
$$

and

$$
g \alpha \beta_{c}^{\circ} d^{4}=[1+(1+c) b] \frac{v}{\rho} \chi \frac{q^{6} d^{4}}{q_{x}^{2}} .
$$

Here $a, b$ and $c$ are the dimensionless parameters introduced in (27). For MBBA $a \simeq \frac{1}{4}$ and $c \simeq \frac{2}{5}$ and one finds that the cross-over point from oscillatory to stationary convection, i.e. the point where $\beta_{\mathrm{c}}^{0}=\beta_{\mathrm{c}}^{\mathrm{s}}$ or equivalently where $\omega_{\mathrm{c}}=0$, takes place for $b \simeq \frac{3}{4}$. For a layer thickness of $5 \mathrm{~mm}$ this corresponds to a magnetic field $H_{\mathfrak{t}} \simeq 550$ gauss. At this point

$$
\beta_{\mathrm{c}}^{0}\left(H_{\mathrm{t}}\right)=\beta_{\mathrm{c}}^{\mathrm{s}}\left(H_{\mathrm{t}}\right) \simeq 2 \beta_{\mathrm{c}}^{0}(H=0) .
$$

Further from (32) it follows that $\omega_{\mathrm{c}}$ reaches its maximum for $b \simeq \frac{1}{4}$ (corresponding to a field strength of roughly 180 gauss) and $\left(\omega_{\mathrm{c}}^{2}\right)_{\max } \simeq 1.4 \omega_{\mathrm{c}}^{2}(H=0)$. The stability diagram of a homeotropic nematic with positive heat diffusion anisotropy in a stabilizing magnetic field is sketched in figure 2 and the behavior of the frequency of the overstable oscillations is represented qualitatively in figure 3 .

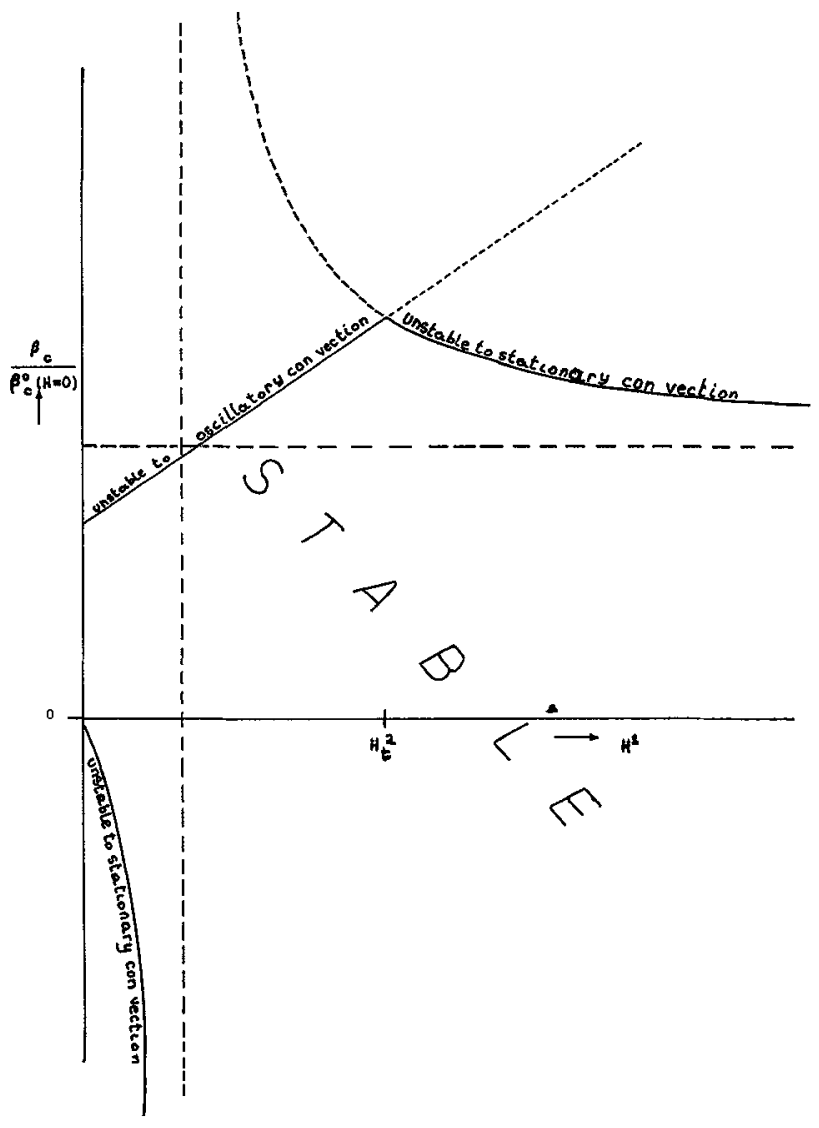

FIG. 2. - Thresholds and instability regions for a homeotropic nematic with positive heat diffusion isotropy in a stabilizing magnetic field.

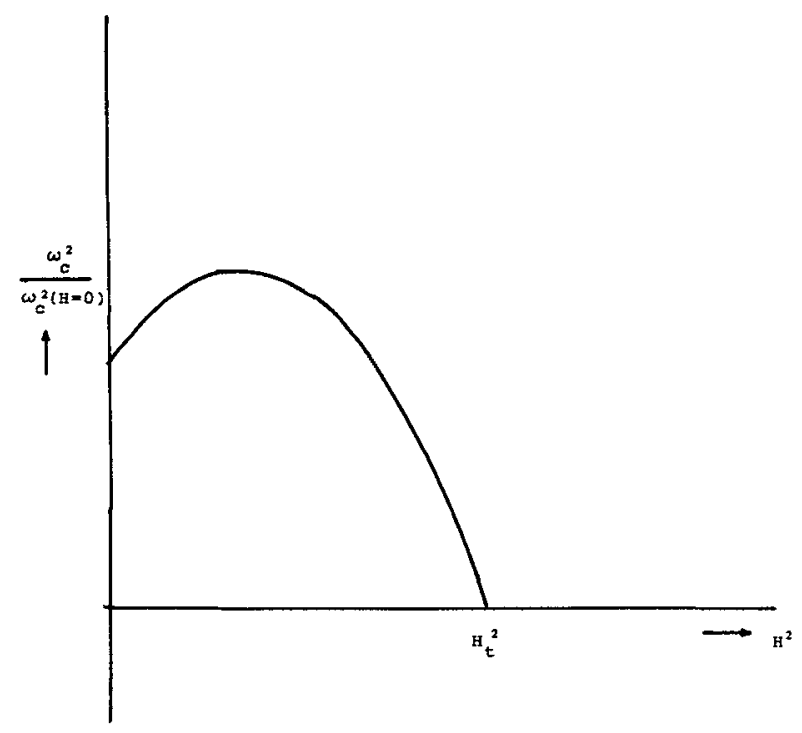

FIG. 3. - The behavior of the frequency of overstable oscillations as a function of a stabilizing magnetic field.

Acknowledgments. - The author is indebted to W. G. Laidlaw and E. Guyon for several useful discussions. This work has been supported by NATO (Grant 806) and interuniversitair Instituut voor Kernwetenschappen (Belgium). 


\section{References}

[1] LekKerkerker, H. N. W., J. Physique Lett. 38 (1977) L-277. [2] Guyon, E., Pieranski, P. and Salan, J., C. R. Hebd. Séan. Acad. Sci. B 287 (1978) 41 and J. Fluid Mech. to appear.

[3] Chandrasekhar, S., Max Planck Festschrift 1958 (Veb. Deutscher Verlag der Wissenschaften, Berlin) 1958, p. 103.

[4] Dubois-Violette, E., C. R. Hebd. Séan. Acad. Sci. B 273 (1971) 923 .
[5] Guyon, E. and Pieranski, P., C. R. Hebd. Séan. Acad. Sci. B 274 (1972) 656.

[6] Pieranski, P., Dubois-Violette, E. and Guyon, E., Phys. Rev. Lett. 30 (1973) 736.

[7] Dubors-Violette, E., Guyon, E. and Pieranski, P., Mol. Cryst. Liq. Cryst. 26 (1974) 193.

[8] Barratt, P. J. and Sloan, D. M., J. Phys. A 9 (1976) 1987. 